\title{
MICA/IL-12: A novel bifunctional protein for killer cell activation
}

\author{
ASHLEE TIETJE $^{1}$, XI YANG $^{2}$, XIANZHONG YU $^{2}$ and YANZHANG WEI ${ }^{2}$ \\ ${ }^{1}$ Division of Science, Southern Wesleyan University, Central, SC 29634; \\ ${ }^{2}$ Department of Biological Sciences, Clemson University, Clemson, SC 29634, USA
}

Received July 20, 2016; Accepted December 5, 2016

DOI: $10.3892 /$ or.2017.5375

\begin{abstract}
Natural killer (NK) cells have the potential to be effective killers of tumor cells. They are governed by inhibitory and activating receptors such as NKG2D, whose ligands are normally upregulated in cells that are stressed, like cancer cells. Advanced cancer cells, however, have ways to reduce the expression of these ligands, leaving them less detectable by NK cells. Along with these receptors, NK cells also require activating cytokines, such as IL-12. A previous study in our laboratory showed that a fusion protein of the extracellular domain of mouse UL-16 binding protein-like transcript 1 (MULT1E) and mouse interleukin 12 (IL-12) can effectively activate mouse NK cells by in vitro assays and in vivo in animal tumor models. The aim of the present study was to expand the concept of developing a novel bifunctional fusion protein for enhanced NK cell activation to human killer cells. The proposed protein combines the extracellular domain of a human NKG2D ligand, MHC class I polypeptide-related sequence A (MICA) and IL-12. It is hypothesized that when expressed by tumor cells, the protein will activate human NK and other killer cells using the NKG2D receptor, and deliver IL-12 to the NK cells where it can interact with the IL-12R and enhance cytotoxicity. The fusion protein, when expressed by engineered tumor cells, indeed activated NK92 cells as measured by an increase in interferon- $\gamma$ (IFN- $\gamma$ ) production and an increase in cytotoxicity of tumor cells. The fusion protein was also able to increase the proliferation of human peripheral blood mononuclear cells (PBMCs) and augment their production of IFN- $\gamma$. This study along with the data from the previous mouse studies suggest that the MICA/IL-12 bifunctional fusion protein represents an effective activator of killer cells for cancer treatment.
\end{abstract}

Correspondence to: Dr Yanzhang Wei, Department of Biological Sciences, Clemson University, 132 Long Hall, Clemson, SC 29634 , USA

E-mail: ywei@clemson.edu

Key words: MICA, IL-12, NKG2D, fusion gene, bifunctional protein, NK cell, immunotherapy

\section{Introduction}

Natural killer (NK) cells are part of the innate immune system and are one of the primary cell types involved in anticancer immunosurveillance. At the onset of neoplastic growth, tumor cells upregulate surface antigens, some of which are ligands for NK cell activating receptors, including NKG2D. Once NK cells are activated, cytotoxic granules are exocytosed into the extracellular space between the NK cell and the target cell and apoptosis of target cells is induced (1). As the cancer progresses, however, advanced tumor cells have found ways to downregulate surface NKG2D ligands. It has been shown that the extracellular domains of MHC class-I related molecule A (MICA), a human NKG2D ligand, can be shed by tumor cells by proteolytic cleavages, thus, preventing activation of cytotoxic immune cells (2). The immune system may also aid in selecting against tumor cells that maintain NKG2D ligand expression (3). Due to these properties, MICA serves as an excellent target for anticancer gene therapy.

Interleukin 12 (IL-12), also known as NK cell stimulatory factor 2, is an activator and initiator of NK cell proliferation. The activity of IL-12 is more efficient than either IL-2 or the IFNs, requiring picomolar instead of nanomolar concentrations (4). In fact, NK cells genetically engineered to express IL-12 in a membrane anchored form by mouse sonic hedgehog C-terminal domain showed reduced ( $>10$-fold) dependency on IL-2 and a significantly prolonged survival time for tumor- bearing mice receiving an intravenous injection of these cells (5). IL-12 has been shown consistently to have potent antitumor activity primarily through the activity of IFN- $\gamma$, the activation of $\mathrm{CD}^{+} \mathrm{T}$ cells, and the inhibition of angiogenesis $(6,7)$.

A previous study in our laboratory showed that a bifunctional fusion protein containing the mouse NKG2D ligand mouse UL-16 binding protein-like transcript 1 (MULT1) and mouse IL-12 can effectively activate mouse NK cells both in vitro and in vivo leading to a reduction in tumor size. It is believed that the enhanced antitumor effect is due to the simultaneous activation of NK cells and other NKG2D expressing killer cells through the MULT1/NKG2D pathway and the IL-12/IL-12R pathway. In addition, MULT1 may also function as a carrier to deliver IL-12 directly to the killer cells. When the MULT1 domain binds to an NKG2D receptor on the surface of an NK cell, the IL-12 may also be held in 
close proximity to IL-12 receptors on the same cell, which will maintain a high level of IL-12 in the tumor microenvironment while providing low systemic IL-12 levels and reduced toxicity (8).

For this work to be translated for clinical use, the protein needs to be adapted for use in humans. The purpose of the present study is to develop a bifunctional fusion protein containing a human NKG2D ligand, MICA and IL-12. Since mouse IL-12 is fully effective in engaging the human IL-12 receptor, a recombinant mouse IL-12 gene is used in this model study (9). This human version of the fusion protein was first examined by transfecting the fusion gene into the human lung carcinoma cell line A549. Stable clones of tumor cells expressing the fusion protein and control IL-12 or MICA proteins were evaluated in a series of in vitro assays to determine their ability to activate the human natural killer cell line NK92 and isolated PBMCs.

\section{Materials and methods}

Cells. The human lung carcinoma cell line A549 (ATCC no. CRM-CCL-185) was cultured in F-12K medium containing $10 \%$ fetal bovine serum (FBS) and $100 \mu \mathrm{g} / \mathrm{ml}$ gentamicin at $37^{\circ} \mathrm{C}$ with $5 \% \mathrm{CO}_{2}$. Human natural killer cell line (CRL-2407) NoGFP-CD16.NK92 (PTA-6967) and the freshly isolated human PBMCs were cultured in $\alpha$-MEM with $2 \mathrm{mM}$ L-glutamine, $1.5 \mathrm{~g} / 1$ sodium bicarbonate, $0.2 \mathrm{mM}$ inositol, $0.1 \mathrm{mM}$ 32-mercaptoethanol, $0.02 \mathrm{mM}$ folic acid, $200 \mathrm{U} / \mathrm{ml}$ recombinant IL-2, $12.5 \%$ horse serum, $12.5 \%$ FBS and $100 \mu \mathrm{g} / \mathrm{ml}$ gentamicin at $37^{\circ} \mathrm{C}$ with $5 \% \mathrm{CO}_{2}$. The blood samples were procured following a GHS IRB approved protocol (CC/ORI 07-02) and the patients consented that their blood materials would be used for research purposes.

Construction of fusion gene and control gene vectors. pcDNA3.1(+)MICA/IL-12. The MICA extracellular domain sequence was amplified from pCMV-SPORT6-MICA (Open Biosystems, Lafayette, CO, USA) using the 5' primer CCCAA GCTTGAGAGGGTGGCGACGTCGGGG, the 3' primer CG GGATCCCTGCCAATGACTCTGAAGCACC and Phusion High-Fidelity DNA polymerase. The 5' primer contains a restriction cut site for HindIII and the 3' primer contains the restriction cut site for BamHI. The PCR fragment was excised and gel purified using a gel purification kit. Double enzyme digestion using HindIII and BamHI was performed on the purified fragment. The plasmid pcDNA3.1(+)MULT1E/mIL-12, created earlier in our laboratory (8), was also digested with HindIII and BamHI to remove the MULT1E and allowing for the insertion of MICA directly upstream of a (GGGS) $)_{3}$ linker and IL-12 in frame. The linearized plasmid was excised and gel purified using a gel purification kit. The enzyme digested MICA PCR fragment was then ligated to the vector creating the new plasmid pcDNA3.1(+)MICA/IL-12 (Fig. 1). The sequence was confirmed using DNA sequencing.

pcDNA3.1(+)IL-12vector. Tomake acontrolIL-12 vector, the IL-12 sequence was amplified from pORF-mIL-12 using the 5' primer CCAAGCTTCCATGGGTCAATCACGCTACCTCC with a HindIII cut site and the 3' primer CCTCGAGCTAG GATCGGACCCTGCAGGG with a XhoI restriction cut site using Phusion High-Fidelity DNA polymerase. The fragment

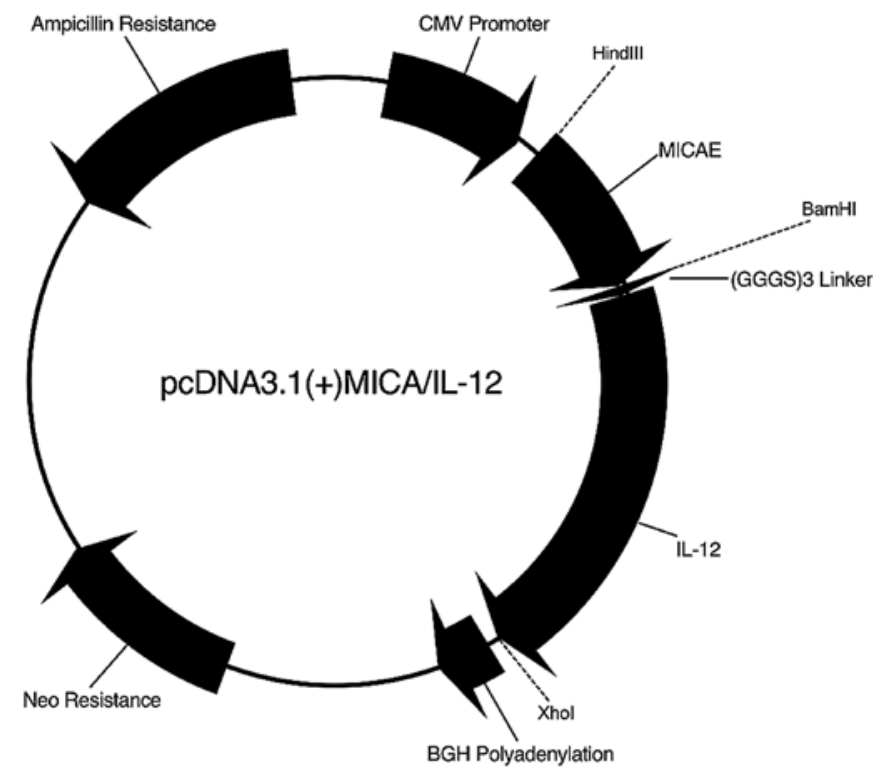

Figure 1. Plasmid pcDNA3.1(+)MICA/IL-12. The MICA extracellular domain sequence was amplified from pCMV-SPORT6-MICA and used to replace MULT1E in the plasmid pcDNA3.1(+)MULT1E/mIL-12 that had been created earlier in our laboratory (1). MICA was inserted directly upstream of a (GGGS) $)_{3}$ linker and IL-12 in frame creating the new plasmid pcDNA3.1(+)MICA/IL-12.

was excised and gel purified using a gel purification kit. Double enzyme digestion was performed on the purified fragment using HindIII and XhoI. pcDNA3.1(+)Neo was digested with HindIII and XhoI allowing for the insertion of IL-12. The fragment was excised and gel purified using a gel purification kit. The IL-12 sequence was then ligated to pcDNA3.1(+)Neo creating the new plasmid pcDNA3.1(+)IL-12. The sequence was confirmed using DNA sequencing.

pcDNA3.1(+) MICA vector. To make a control MICA vector encoding a secretable form of the extracellular domain of MICA, the extracellular MICA sequence was amplified from pcDNA3.1(+)MICA/IL-12. The 5' primer was the same used for the construction of pcDNA3.1(+)MICA/IL-12 and the 3' primer (CCTCGAGCTACTGCCAATGACTCT) included a stop codon and the restriction site for XhoI. PCR was performed to amplify MICA using Phusion High-Fidelity DNA polymerase. The fragment was excised and gel purified using a gel purification kit. Double enzyme digestion was performed on the purified fragment using HindIII and XhoI. pcDNA3.1(+)Neo was digested with HindIII and XhoI allowing for the insertion of MICA. The fragment was excised and gel purified using a gel purification kit. The MICA sequence was then ligated to the pcDNA3.1(+)Neo creating the new plasmid pcDNA3.1(+)MICA. The sequence was confirmed using DNA sequencing.

Cell transfection. A549 cells were transfected with either pcDNA3.1(+)MICA/IL-12, pcDNA3.1(+)IL-12 or pcDNA3.1(+) MICA using Lipofectamine as directed by the manufacturer. To obtain stable clones, the transfected A549 cells were cultured in medium containing $250 \mu \mathrm{g} / \mathrm{ml}$ of G418. Drugresistant clones were collected and subcultured in the presence of the appropriate drug. 
Proliferation assay. To determine if the selected clones grow at the same rate as the untransfected parental A549 cells, $0.05 \times 10^{6}$ cells/well were plated and cultured on 96-well plates in triplicates and counted every day for 5 days. At each time point, CellTiter 96 Aqueous non-radioactive cell proliferation assay (Promega, Madison, WI, USA) was used following the manufacturer's instructions to determine the relative cell count. Clones were compared using a two-way ANOVA with the Tukey's post-test.

$R T-P C R$. Total RNA was extracted from each clone using an RNeasy Plus Mini kit following the manufacturer's directions. RT-PCR was run with $1 \mu \mathrm{g}$ of the total RNA using the Phusion RT-PCR kit following the manufacturer's directions. To amplify a 177-bp segment from MICA the 5' primer CCTTG GCCATGAACGTCAGG and the 3' primer CCTCTGAGG CCTCGCTGCG were used. To amplify an 805 bp sequence of IL-12 the 5' primer GGGTGATGGGCTATCTGAGC and the 3' primer AACTTGAGGGAGAAGTAGGAATGG were used. To amplify a $1.2 \mathrm{~kb}$ portion of the MICA/IL-12 fusion gene containing $446 \mathrm{bp}$ of $\mathrm{MICA}$, the (GGGS) ${ }_{3}$ linker, and $708 \mathrm{bp}$ of IL-12 the 5' primer CCTTGGCCATGAACGTCAGG and the 3 ' primer GGGAGTCCAGTCCACCTCTA were used. All reactions included the GAPDH housekeeping gene using the 5' primer ATGACATCAAGAAGGTGGTG and the 3' primer CATACCAGGAAATGAGCTTG.

Detection of secreted protein. Cells of different clones were plated at $2 \times 10^{6}$ cells in 6 -well plates with normal growth media on day one. On day two, the media was removed, the cells were rinsed with phosphate-buffered saline (PBS) and serum-free F-12K was added. On day three, the serum-free RPMI was removed and centrifuged at $1000 \mathrm{x} g$ for $15 \mathrm{~min}$. The supernatant was then tested using an appropriate immunofluorescence or ELISA protocol.

To detect the presence of MICA/IL-12, 96-well plates were coated with $2 \mu \mathrm{g} / \mathrm{ml} \mathrm{rhNKG} 2 \mathrm{D} / \mathrm{Fc}$ chimera in carbonate coating buffer and incubated overnight at $4^{\circ} \mathrm{C}$. The plates were rinsed with PBS and blocked with $10 \%$ FBS for $1.5 \mathrm{~h}$. After washing three times with PBS, $100 \mu \mathrm{l}$ of the cell supernatant was added to the plate in triplicates. The samples were incubated at room temperature for $3 \mathrm{~h}$. After removing the samples and washing the plates three times with PBS, $1 \mu \mathrm{g} / \mathrm{ml} \mathrm{PE} \mathrm{rat}$ anti-mouse IL-12 (p40/p70) in blocking solution was added to the plates. The plates were then incubated for $1.5 \mathrm{~h}$ at room temperature. After washing five times with PBS, the plates were read with a BioTek Synergy H1 hybrid reader. Clones were compared using a one-way ANOVA with the Tukey's post-test. Corrected RFU is calculated by subtracting background RFU from each value.

To detect the presence of secreted IL-12 from the cells, a mIL-12 p70 Ready-SET-Go ELISA kit was used following the manufacturer's directions. Plates were read with a BioTek EON microplate reader. Clones were compared using a one-way ANOVA with the Tukey's post-test.

NK92 and A549 co-culture. NK92 cells were plated with A549 cells or clones expressing the fusion protein, IL-12, or MICA on a 96-well plate at a ratio of 10:1 in $250 \mu \mathrm{l} \mathrm{NK}$ media and incubated for $48 \mathrm{~h}$. After the incubation, $100 \mu \mathrm{l}$ of media was removed and analyzed for the presence of IFN- $\gamma$ using human IFN- $\gamma$ ELISA reagent kit (Thermo Fisher Scientific, Waltham, MA, USA) following the manufacturer's directions. The rest of the media were removed from the cells, the NK suspension cells were carefully rinsed off, and $100 \mu \mathrm{l}$ fresh NK media was added. The remaining tumor cell number was determined using CellTiter 96 AQueous non-radioactive cell proliferation assay (Promega) following the manufacturer's instructions. Clones were compared using a one-way ANOVA with the Tukey's post-test.

Primed NK92 cytotoxicity. The A549 clones were plated on a 6 -well plate in $5 \mathrm{ml} \mathrm{NK}$ media at a density of $0.5 \times 10^{6}$ cells/well and incubated for $48 \mathrm{~h}$. After the incubation, the supernatant was collected from these cells and used to plate $4 \times 10^{5} \mathrm{NK} 92$ cells on a 6 -well plate. After a $48 \mathrm{~h}$ priming period, the NK92 cells were removed and plated on a $96-$ well plate at a density of $5 \times 10^{4}$ cells/well with $0.5 \times 10^{4}$ parental A549 cells and incubated for $24 \mathrm{~h}$. After the incubation, the media were removed from the cells and the wells were carefully washed four times to remove the non-adherant NK92 cells. A total of $100 \mu \mathrm{l}$ fresh NK media was added. Cell proliferation was determined using CellTiter 96 AQueous non-radioactive cell proliferation assay (Promega) following the manufacturer's instructions. Clones were compared using a one-way ANOVA with the Tukey's post-test. Corrected OD is calculated by subtracting background OD from each value.

Preparation of human PBMCs. PBMCs were isolated from leukapheresis product using the Ficoll-Paque method. Briefly, the blood product was mixed 1:4 with PBS and loaded on top of $30 \mathrm{ml}$ lymphocyte separation media and was centrifuged for $15 \mathrm{~min}$. The PBMC layer was then removed and rinsed with PBS. ACK lysing buffer was then used to lyse any remaining RBCs. After a final PBS rinse, cells were counted and used.

Supernatant transfer to PBMC. The A549 clones were plated on a 6 -well plate in $1.5 \mathrm{ml}$ PBMC media at a density of $0.6 \times 10^{6}$ cells/well and incubated for $48 \mathrm{~h}$. After the incubation, the supernatant was collected from these cells and used to plate $2 \times 10^{5}$ freshly isolated PBMCs on a 96-well plate and the cells were incubated for 10 days. After the incubation, $100 \mu \mathrm{l}$ of media was removed and analyzed for the presence of IFN- $\gamma$ using human IFN- $\gamma$ ELISA reagent kit (Thermo Fisher Scientific) following the manufacturer's directions. The rest of the media was removed from the cells and $100 \mu \mathrm{l}$ fresh PBMC media were added. Cell proliferation was determined using CellTiter 96 AQueous non-radioactive cell proliferation assay (Promega) following the manufacturer's instructions. Clones were compared using a one-way ANOVA with the Tukey's post-test.

Statistical analysis. GraphPad software was used to plot graphs and run statistics. The significance was represented as $\mathrm{P}<0.05, \mathrm{P}<0.01$ or $\mathrm{P}<0.001$.

\section{Results}

Construction of expression vectors. To construct the plasmid containing the MICA/IL-12 fusion gene, the MICA 


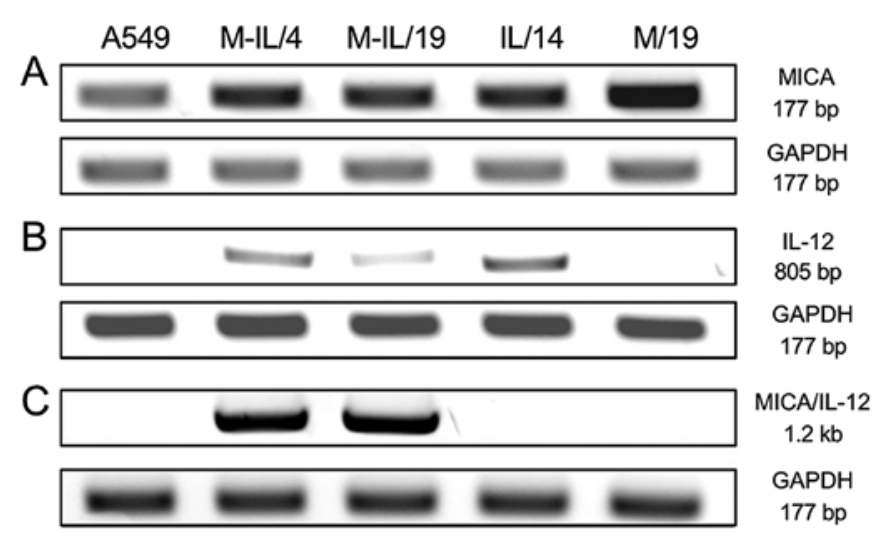

Figure 2. RT-PCR analysis of A549 clones with transgenes. Total RNAs were isolated from each clone and reverse transcription was used to create cDNA. Primers were used to amplify a 177-bp portion of the extracellular domain of MICA (A), a 805-bp portion of IL-12 (B), and a $1.2 \mathrm{~kb}$ portion of the MICA/IL-12 fusion gene containing 446 bp of MICA, the (GGGS) $)_{3}$ linker, and 708 bp of IL-12 using PCR.

extracellular domain cDNA sequence was amplified from pCMV-SPORT6-MICA and used to replace MULT1E in the plasmid pcDNA3.1(+)MULT1E/mIL-12 that had been constructed earlier in our laboratory (1). MICA was inserted directly upstream of a (GGGS) $)_{3}$ linker, allowing flexibility, and IL-12 in frame creating the new plasmid pcDNA3.1(+) MICA/IL-12 (Fig. 1). The IL-12 control plasmid, pcDNA3.1(+) IL-12, was constructed by amplifying the IL-12 sequence from pORF-mIL-12 and ligating it into the pcDNA3.1(+) vector. Since the fusion protein is secretable and only contains the extracellular domain of MICA, PCR was used to amplify MICA from pcDNA3.1(+)MICA/IL-12 and add a stop codon at the end of the extracellular domain. Since MICA is a type I transmembrane protein and the signal sequence is conserved, the resulting protein should be transported outside the cell (10).

Transfection and stable clone selection. A549 cells were transfected with plasmids pcDNA3.1(+)MICA/IL-12 (Fig. 1), pcDNA3.1(+)MICA, or pcDNA3.1(+)IL-12, respectively, using Lipofectamine. Multiple clones from each transfection that were geneticin resistant were selected. RT-PCR and ELISA analysis (see below) were used to screen these clones and clones M-IL/4 and M-IL/19 were selected for fusion protein MICA/IL-12, clone IL/14 for IL-12 and clone M/19 for MICA. An in vitro cell proliferation assay showed that all clones grow at a similar rate (data not shown).

Stable clones express the fusion protein. The fusion protein expression was first examined by reverse transcription-PCR (RT-PCR) on total RNA samples collected from the clones using three pairs of primers. The first pair was specific to a $177 \mathrm{bp}$ portion of the extracellular domain of MICA. The second pair amplified an 805 bp sequence of IL-12. The final primer pair amplified a $1.2 \mathrm{~kb}$ portion of the MICA/IL-12 fusion gene containing $446 \mathrm{bp}$ of MICA, the (GGGS) ${ }_{3}$ linker, and 708 bp of IL-12. All cells, including the A549 control cells and IL/14, contain mRNA transcripts for MICA (Fig. 2A). The fusion gene clones, along with the IL/14 clone contain mRNA transcripts for IL-12 (Fig. 2B). Only clones M-IL/4
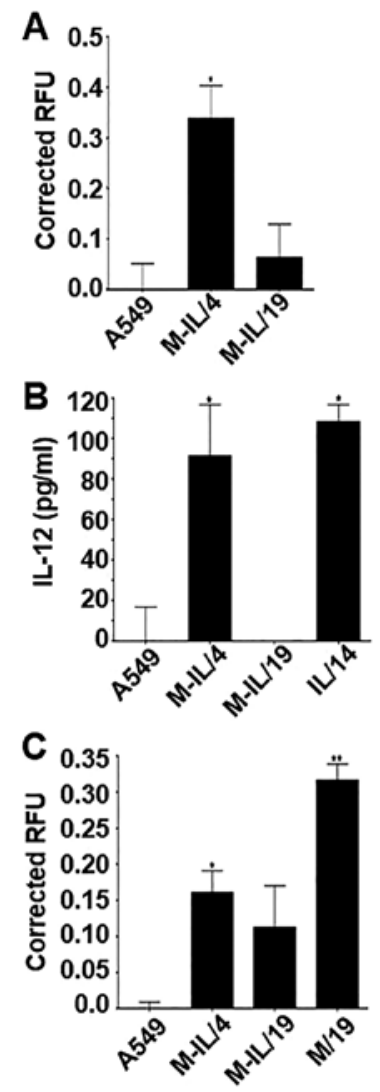

Figure 3. Detection of secreted transgene products from A549 clones Supernatant from each clone was collected for ELISA analysis. (A) The fusion gene was detected by coating the plate with $\mathrm{rhNKG} 2 \mathrm{D} / \mathrm{Fc}$ chimera, adding the supernatant, and using anti-mIL-12 antibody conjugated with PE as the detecting antibody. (B) IL-12 was detected using an mIL-12 ELISA kit. (C) MICA was detected by coating the plate with rhNKG2D/Fc chimera, adding the supernatant, and using anti-hMICA phycoerythrin conjugated as the detecting antibody. Corrected RFU is calculated by subtracting background RFU from each value. Clones were compared using a one-way ANOVA with the Tukey's post-test. ${ }^{*} \mathrm{P}<0.05$ and ${ }^{* *} \mathrm{P}<0.01$.

and M-IL/19 contain mRNA transcripts for the MICA/IL-12 fusion gene (Fig. 2C).

A sandwich ELISA was used to detect the secretion of the transgene products. The rhNKG2D/Fc chimera was used to coat the plate and anti-mouse IL-12 conjugated with PE was used as the detecting antibody. Clone M-IL/4 produced significant levels of the MICA/IL-12 fusion protein and successfully released it into the supernatant. Clone M-IL/19 did not produce detectable amounts of the fusion protein (Fig. 3A). ELISA was also performed to detect IL-12 or MICA separately. Clones M-IL/4 and IL/14 both produced similar amounts of IL-12 (Fig. 3B). M-IL/19 produced no detectable levels of IL-12. The secreted form of MICA was detected at significant levels in clones M-IL/4 and M/19 (Fig. 3C).

MICA/IL-12 activates NK92 cells. Human NK92 cells were co-cultured with the A549 cells and clones M-IL/4, M-IL/19, $\mathrm{I} / 14$, or $\mathrm{M} / 19$ for $48 \mathrm{~h}$. To determine if the proteins produced by these clones could activate the NK92 cells, two assays were performed. First, $100 \mu \mathrm{l}$ of the media was removed and screened using an IFN- $\gamma$ ELISA. The NK92 cells co-cultured with clones M-IL/4, IL/14 and M/19 showed significantly higher IFN- $\gamma$ production than NK92 cells co-cultured with 

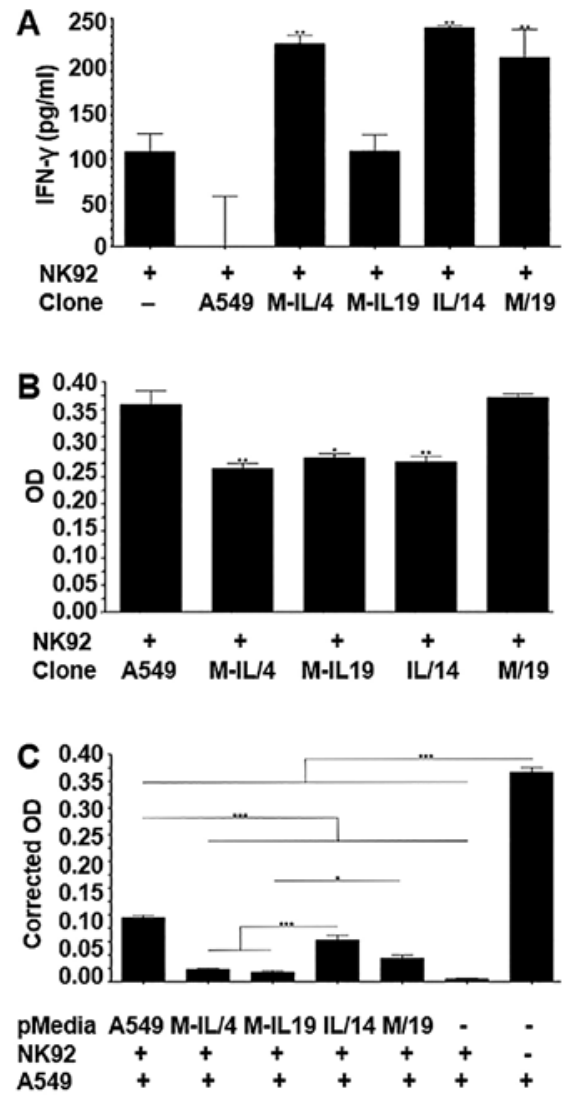

Figure 4. NK92 cell activation. NK92 cells were co-cultured with different clones in a 96 well plate at a ratio of 10:1 and incubated for $48 \mathrm{~h}$. (A) After the incubation, media was removed and analyzed for the presence of IFN- $\gamma$ using ELISA. (B) The NK92 cell cytotoxicity was determined by measuring the proliferation of the tumor cells using CellTiter 96 AQueous non-radioactive cell proliferation assay following the manufacturer's instructions. (C) NK92 cells primed with media collected from A549 clones were co-cultured with parental A549 cells at a ratio of 10:1 for $24 \mathrm{~h}$. The amount of A549 cells remaining after the incubation time was again measured using CellTiter 96 AQueous non-radioactive cell proliferation assay (Promega) following the manufacturer's instructions. Corrected OD is calculated by subtracting background $\mathrm{OD}$ from each value. Clones were compared using a one-way ANOVA with the Tukey's post-test. ${ }^{*} \mathrm{P}<0.05,{ }^{* *} \mathrm{P}<0.01$ and ${ }^{* * * *} \mathrm{P}<0.001$.

A549 parental cells. M-IL/19 was not able to significantly increase IFN- $\gamma$ production (Fig. 4A).

The second assay measured the primed NK92 cell cytotoxicity towards the tumor cell clones. After removing the supernatants from the co-cultures, the wells were gently rinsed five times with PBS to remove the remaining suspension NK92 cells and leave behind the adherent tumor cells. The remaining cells were then measured using an MTS cell proliferation assay. There were significantly fewer M-IL/4, M-IL/19 and IL/14 cells when compared to the A549 parental cells. There was no significant difference in the number of M/19 cells compared to the A549 parental cells (Fig. 4B).

We assessed whether the pre-activation or priming of NK92 cells with supernatant collected from the clones expressing the fusion protein, IL-12, or MICA can enhance the killing of parental A549 cells. A549 parental cells and the clones $\mathrm{M}-\mathrm{IL} / 4, \mathrm{M}-\mathrm{IL} / 19$, IL/14 or M/19 were cultured in NK media for $48 \mathrm{~h}$ before collecting the supernatant. NK92 cells were then plated in the collected supernatants and
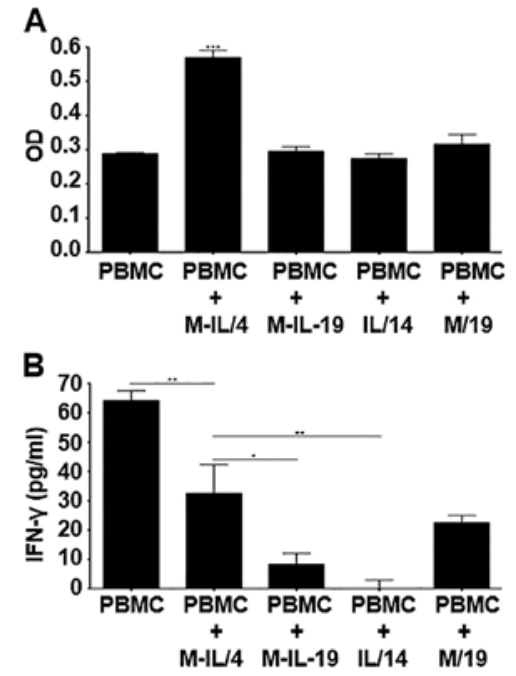

Figure 5. PBMC activation. Supernatant collected from A549 cells expressing the fusion protein, IL-12, or MICA was used to activate isolated human PBMCs. After the incubation the relative cell proliferation was determined using CellTiter 96 AQueous non-radioactive cell proliferation assay (A) and the media was removed and analyzed for the presence of IFN- $\gamma(\mathrm{B})$. Clones were compared using a one-way ANOVA with the Tukey's post-test. ${ }^{*} \mathrm{P}<0.05$, ${ }^{* *} \mathrm{P}<0.01$ and ${ }^{* * *} \mathrm{P}<0.001$.

allowed to incubate for $48 \mathrm{~h}$. These primed NK92 cells were then plated with parental A549 cells at a ratio of 10:1 and incubated for $24 \mathrm{~h}$. At the end of the incubation, the culture media were removed and the wells were gently rinsed five times to remove the suspension NK92 cells and leave behind the adherent A549 cells. The remaining cells were measured using an MTS cell proliferation assay. Although all NK92 cells, whether primed or not, were able to significantly reduce the tumor cell number compared to the control, M-IL/4 and M-IL/19 primed NK92 cells were able to kill significantly more tumor cells than either A549, IL-4, or M/19 primed NK92 cells. Notably, unprimed NK92 cells were the most effective killers of A549 cells (Fig. 4C).

MICA/IL-12 activates human PBMCs. Human peripheral blood mononuclear cells (PBMCs) were isolated from leukapheresis product using the Ficoll-Paque method. These cells were then cultured with supernatants collected from A549 cells or clones M-IL/4, M-IL/19, M/19, or IL/14 for 10 days. To determine if the supernatants could promote PBMC proliferation, an MTS cell proliferation assay was performed. Only supernatant collected from clone M-IL/4 that expresses the fusion protein at a relatively high level was able to significantly increase PBMC cell number (Fig. 5A). An IFN- $\gamma$ ELISA was also used to detect killer cell activation. Clone M-IL/4 was also able to induce a significantly higher production of IFN- $\gamma$ when compared to clones M-IL/19 and IL/14. Clone M/19 also induced production of a large amount of IFN- $\gamma$ (Fig. 5B). Interestingly, PBMCs without co-culture expressed the highest level of IFN- $\gamma$.

\section{Discussion}

Since NK cells play an important role in tumor immunosurveilance, many strategies have been employed to harness their 
ability to prevent neoplastic growth. In the present study we developed a bifunctional fusion gene encoding MICA/IL-12, and hypothesized that the bifunctional protein, when expressed by tumor cells, would effectively activate NK cells and other NKG2D expressing killer cells in the tumor environment and increase cytotoxicity.

RT-PCR indicates that all clones contained mRNA for MICA (Fig. 2A). This is not surprising since MICA is commonly expressed in lung carcinomas (11). IL-12 mRNA is detected in the two fusion gene clones (M-IL/4 and M-IL/19) and the IL-12 clone (IL/14) (Fig. 2B). Finally, a portion of the MICA/IL-12 fusion protein including the linker segment was detected only in the fusion gene clones M-IL/4 and M-IL/19. The data indicate that the clones have in fact taken up the transgenes and transcription of the genes of interest can be detected.

It is important to demonstrate that the fusion protein is produced and being released from the cell into the tumor microenvironment where it can interact with killer cells. To test this, supernatants were collected from all the clones together with A549 cells and screened using ELISA. As Fig. 3 shows, the engineered proteins are produced by the respective clones and released from the cells into the supernatants. Clone M-IL/4 produces a relatively high level of fusion protein MICA/IL-12, but it is not detectable in the supernatant collected from M-IL/19 (Fig. 3A). Because there was a clear band detected after RT-PCR indicating that M-IL/19 does contain the fusion gene, it is possible that the protein amount produced by this clone is beneath the detection threshold of the methods used. Both clones M-IL/4 and IL/14 produce significant levels of IL-12. It should also be noted that the amount of IL-12 detected in the clone M-IL/4 supernatant is similar to the amount of IL-12 detected in the clone IL/14 supernatant. This indicates that clone IL/14 is a good control for this experiment (Fig. 3B). As expected, significant levels of MICA were detected in the supernatants from clones M-IL/4 and $M / 19$. Since there is not a significant difference between the MICA levels of clone M-IL/4 and M/19, clone M/19 is a good control for this experiment (Fig. 3C).

To determine if fusion protein MICA/IL-12 is functional and has enhanced ability to activate killer cells, the human natural killer cell line NK92, was co-cultured with A549 cells and clones M-IL/4, M-IL/19, IL/14 and M/19 for $48 \mathrm{~h}$ at a ratio of $10: 1$. The amount of IFN- $\gamma$ produced by the NK92 cells was evaluated with ELISA to determine the level of NK cell activation. The NK92 cells co-cultured with clones M-IL/4, IL/14 and $M / 19$ showed significantly higher IFN- $\gamma$ production than NK92 cells co-cultured with A549 parental cells. Clone IL/14 was also able to increase IFN- $\gamma$ production significantly compared to NK92 cells that were not cultured with any A549 cells. M-IL/19 was not able to significantly increase IFN- $\gamma$ production compared to NK92 cells alone (Fig. 4A). M-IL/4 stimulated production at similar levels of IFN- $\gamma$ as clone IL/14, which is expected because they both have similar IL-12 expression levels as shown in the IL-12 ELISA (Fig. 3B). It is interesting that NK92 cells co-cultured with A549 parental cells produced much less IFN- $\gamma$ when compared with NK92 cells that were not co-cultured with any cells. This is possibly due to either the production of or the shedding of inhibitory ligands from A549 tumor cells into the supernatant, a phenomenon commonly seen in tumors, which can reduce the activation of the NK92 cells by engaging the inhibitory receptors, such as NKG2A/B, KIR2DL4 and ILT-2 (12-16). The results not only confirm the notion that the activation of $\mathrm{NK}$ cells and other killer cells depends on the balance of inhibitory receptors and activating receptors, but also demonstrate that the fusion protein MICA/IL-12 can change the balance towards activation.

To determine if the fusion protein could enhance NK92 cytotoxicity toward the tumor cells, the number of A549 cells remaining after the co-culture was measured. There were significantly fewer cells of clones M-IL/4, M-IL/19 and IL/14 when compared to the A549 parental cells or clone M/19 cells (Fig. 4B). Although the cytotoxicities among clones M-IL/4, M-IL/19 and IL/14 are not statistically different, clone M-IL/4 shows a slightly higher activity. This indicates that clone M-IL/19 does produce some MICA/IL-12 even though it was undetectable using ELISA (Fig. 3C). This suggests that when compared to a larger amount of IL-12, even a small amount of the fusion protein can produce similar cytotoxic results. This is also interesting because clone M-IL/19 was not able to increase IFN- $\gamma$ production of the NK92 cells whereas M-IL/4 and IL/14 did (Fig. 4A). This suggests that the addition of the MICA portion of the protein increases the cytotoxic activity of the NK92 cells.

One of the current hurdles for gene therapy is the low efficiency of gene delivery (17). With this in mind, it is important to determine if MICA/IL-12 can also activate NK92 cells to kill A549 cells that do not express the protein. NK92 cells that were primed for $48 \mathrm{~h}$ with the supernatants from A549 cells and clones of M-IL/4, M-IL/19, IL/14 and M/19 were co-cultured with parental A549 cells at a ratio of 10:1 and incubated for $24 \mathrm{~h}$. After the removal of the suspension NK92 cells, the remaining tumor cells were measured using an MTS cell proliferation assay. All NK92 cells, whether primed or not, were able to significantly reduce the A549 cell number compared to the control without NK92 cells. This is not a surprise as these cells are highly cytolytic. Both clones M-IL/4 and M-IL/19 primed NK92 cells were able to kill significantly more A549 cells than either A549, IL-4, or M/19 primed NK92 cells. This suggests that priming with MICA/IL-12 is more effective than priming with either IL-12 or MICA alone, even when the amount of MICA/IL-12 is small as seen in clone M-IL/19. Notably, the unprimed NK92 cells killed significantly more A549 cells than all other NK92 cells except the NK92 cells primed with MICA/IL-12 (Fig. 4C). This suggests that MICA/IL-12 expression, even in small amounts, was able to overcome the inhibitory signals from A549 cells during NK92 priming.

Although the NK92 cell line is a good model for human NK cells, it does not give a complete picture of the in vivo effects of fusion protein MICA/IL-12. To better understand how MICA/IL-12 will behave in a patient, further experiments were conducted using human PBMCs isolated from leukapheresis products. Supernatants collected from clones M-IL/4, M-IL/19, IL/14, or M/19 were used to treat PBMCs for 10 days and then a relative cell number was determined. Only supernatant from clone M-IL/4, that expresses the highest level of the fusion protein, is able to significantly increase PBMC cell number (Fig. 5A), indicating that MICA/IL-12 is able to 
either increase proliferation and/or increase sustainability of these cells.

An IFN- $\gamma$ ELISA was also used to detect NK and other killer cell activation in PBMC culture. As Fig. 5B shows, supernatant from clone M-IL/4 stimulates PBMCs to produce the most IFN- $\gamma$, although supernatant from clone $\mathrm{M} / 19$ also stimulates PBMCs to produce relatively high levels of IFN- $\gamma$. M-IL/4 induced a significantly higher production of IFN- $\gamma$ when compared to M-IL/19 and IL/14 (Fig. 5C).

An interesting observation from the present study is that the IFN- $\gamma$ production by unprimed PBMCs is significantly higher than those primed with a clone. This, again, is possibly due to either the production of or the shedding of inhibitory ligands into the supernatant by the tumor cells (12-15). This effect is more pronounced with the PBMCs compared to NK92 cells (Fig. 4A) as the IFN- $\gamma$ production by unprimed PBMCs is higher than that of PBMCs primed with supernatants from any of the tumor cell clones (Fig. 5B), while IFN- $\gamma$ production by NK92 cells primed with clones M-IL/4, IL/14 or M/19 is higher than that of the unprimed NK92 cells. This is probably due to the larger repertoire of PBMC inhibitory receptors. Although the fusion protein MICA/IL-12 did not completely recover the inhibitory effects as it does in NK92 culture, it is much more effective than IL-12 or MICA alone.

Another interesting observation seen when comparing the NK92 and the PBMC results is the effectiveness of the IL/14 and M/19 clones. In the experiments conducted using the NK92 cells, the clone that produced only IL-12 (IL/14) performed better than the clone that produced only MICA (M/19). The IL-12 was able to initiate production of more IFN- $\gamma$ by NK92 cells and resulted in more cell death during the co-culture compared to M/19 (Fig. 4A and B). However, in the PBMC experiments, clone $\mathrm{M} / 19$ initiated the production of more IFN- $\gamma$ by NK92 cells than was initiated by clone IL/14 (Fig. 5B). This suggests that the IL-12 may be a more effective activator of NK cells, but the MICA can activate other killer cell types within the heterogeneous PBMC population. The fusion protein, however, consistently outperforms both individual components when tested separately.

In conclusion, a fusion protein containing the extracellular domain of the NKG2D ligand MICA and IL-12 was successfully created and can be stably expressed by A549 tumor cells. The fusion protein can increase the production of IFN- $\gamma$ by NK92 cells and also increase the cytolytic activity of these cells toward A549 cells expressing MICA/IL-12. The bystander effect was also demonstrated when MICA/IL-12 primed NK92 cells were able to kill parental A549 cells significantly more than NK92 cells primed with either IL-12 or MICA alone. This result was also an indication that the activation by MICA/IL-12 was able to overcome inhibitory signals by the A549 cells. Preliminary studies also indicate that MICA/IL-12 can increase proliferation and/or sustainability of isolated human PBMCs. The data suggest that MICA/IL-12 is effective at augmenting the IFN- $\gamma$ production of cells cultured with soluble inhibitory factors produced by A549 cells. Although the study is preliminary, the data, along with the data from the previous mouse studies suggest that the MICA/IL-12 bifunctional fusion protein is an effective activator of killer cells for cancer treatment.

\section{References}

1. Berke G: The binding and lysis of target cells by cytotoxic lymphocytes: Molecular and cellular aspects. Annu Rev Immunol 12: 735-773, 1994.

2. Salih HR, Rammensee H-G and Steinle A: Cutting edge: Downregulation of MICA on human tumors by proteolytic shedding. J Immunol 169: 4098-4102, 2002.

3. Dunn GP, Old LJ and Schreiber RD: The three Es of cancer immunoediting. Annu Rev Immunol 22: 329-360, 2004.

4. Robertson MJ, Soiffer RJ, Wolf SF, Manley TJ, Donahue C, Young D, Herrmann SH and Ritz J: Response of human natural killer (NK) cells to NK cell stimulatory factor (NKSF): Cytolytic activity and proliferation of NK cells are differentially regulated by NKSF. J Exp Med 175: 779-788, 1992.

5. Zhu L, Zhao Z, Wei Y, Marcotte W Jr, Wagner TE and Yu X: An IL-12/Shh-C domain fusion protein-based IL-12 autocrine loop for sustained natural killer cell activation. Int J Oncol 41: 661-669, 2012.

6. Brunda MJ, Luistro L, Warrier RR, Wright RB, Hubbard BR, Murphy M, Wolf SF and Gately MK: Antitumor and antimetastatic activity of interleukin 12 against murine tumors. J Exp Med 178: 1223-1230, 1993.

7. Voest EE, Kenyon BM, O'Reilly MS, Truitt G, D'Amato RJ and Folkman J: Inhibition of angiogenesis in vivo by interleukin 12 . J Natl Cancer Inst 87: 581-586, 1995.

8. Tietje A, Li J, Yu X and Wei Y: MULT1E/mIL-12: A novel bifunctional protein for natural killer cell activation. Gene Ther 21: 468-475, 2014.

9. Schoenhaut DS, Chua AO, Wolitzky AG, Quinn PM, Dwyer CM, McComas W, Familletti PC, Gately MK and Gubler U: Cloning and expression of murine IL-12. J Immunol 148: 3433-3440, 1992.

10. Halenius A, Gerke C and Hengel H: Classical and non-classical MHC I molecule manipulation by human cytomegalovirus: So many targets-but how many arrows in the quiver? Cell Mol Immunol 12: 139-153, 2015.

11. Groh V, Rhinehart R, Secrist H, Bauer S, Grabstein KH and Spies T: Broad tumor-associated expression and recognition by tumor-derived gamma delta T cells of MICA and MICB. Proc Natl Acad Sci USA 96: 6879-6884, 1999.

12. Baltz KM, Krusch M, Baessler T, Schmiedel BJ, Bringmann A, Brossart P and Salih HR: Neutralization of tumor-derived soluble glucocorticoid-induced TNFR-related protein ligand increases NK cell anti-tumor reactivity. Blood 112: 3735-3743, 2008.

13. Liu C, Yu S, Zinn K, Wang J, Zhang L, Jia Y, Kappes JC, Barnes S, Kimberly RP, Grizzle WE, et al: Murine mammary carcinoma exosomes promote tumor growth by suppression of NK cell function. J Immunol 176: 1375-1385, 2006.

14. Buggins AG, Milojkovic D, Arno MJ, Lea NC, Mufti GJ, Thomas NS and Hirst WJ: Microenvironment produced by acute myeloid leukemia cells prevents $\mathrm{T}$ cell activation and proliferation by inhibition of NF-kappaB, c-Myc, and pRb pathways. J Immunol 167: 6021-6030, 2001.

15. Urosevic M and Dummer R: HLA-G and IL-10 expression in human cancer--different stories with the same message. Semin Cancer Biol 13: 337-342, 2003.

16. Maki G, Klingemann HG, Martinson JA and Tam YK: Factors regulating the cytotoxic activity of the human natural killer cell line, NK-92. J Hematother Stem Cell Res 10: 369-383, 2001.

17. Jin L, Zeng X, Liu M, Deng Y and He N: Current progress in gene delivery technology based on chemical methods and nanocarriers. Theranostics 4: 240-255, 2014. 